\title{
Long non-coding RNA GAS5 acts as proliferation "brakes" in CD133+ cells responsible for tumor recurrence
}

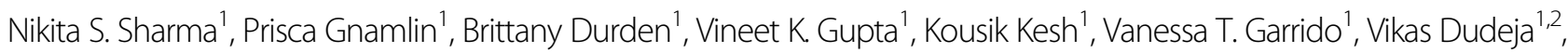 \\ Ashok Saluja ${ }^{1,2}$ and Sulagna Banerjee ${ }^{1,2}$
}

\begin{abstract}
Presence of quiescent, therapy evasive population often described as cancer stem cells (CSC) or tumor initiating cells (TIC) is often attributed to extreme metastasis and tumor recurrence. This population is typically enriched in a tumor as a result of microenvironment or chemotherapy induced stress. The TIC population adapts to this stress by turning on cell cycle arrest programs that is a "fail-safe" mechanism to prevent expansion of malignant cells to prevent further injury. Upon removal of the "stress" conditions, these cells restart their cell cycle and regain their proliferative nature thereby resulting in tumor relapse. Growth Arrest Specific 5 (GAS5) is a long-non-coding RNA that plays a vital role in this process. In pancreatic cancer, CD133+ population is a typical representation of the TIC population that is responsible for tumor relapse. In this study, we show for the first time that emergence of CD133+ population coincides with upregulation of GAS5, that reprograms the cell cycle to slow proliferation by inhibiting GR mediated cell cycle control. The CD133+ population further routed metabolites like glucose to shunt pathways like pentose phosphate pathway, that were predominantly biosynthetic in spite of being quiescent in nature but did not use it immediately for nucleic acid synthesis. Upon inhibiting GAS5, these cells were released from their growth arrest and restarted the nucleic acid synthesis and proliferation. Our study thus showed that GAS5 acts as a molecular switch for regulating quiescence and growth arrest in CD133+ population, that is responsible for aggressive biology of pancreatic tumors.
\end{abstract}

\section{Introduction}

Aggressiveness of a tumor has been correlated with the presence of a population of slow-cycling, treatment refractory and extremely metastatic cells. Accumulating evidence shows that this population is typically enriched in a tumor in response to microenvironmental and/or chemotherapy induced stress. Recent research has attributed this enrichment to senescence associated

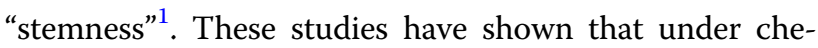
motherapeutic or microenvironmental stress like hypoxia

\footnotetext{
Correspondence: Sulagna Banerjee (Sulagna.Banerjee@med.miami.edu)

${ }^{1}$ Department of Surgery, Miller school of Medicine, University of Miami, Miami, FL, USA

${ }^{2}$ Sylvester Comprehensive Cancer Center, University of Miami, Miami, FL, USA These authors contributed equally: Nikita S Sharma, Prisca Gnamlin
}

or nutrient deprivation, a population of cells specifically respond to the induced stress by triggering a cell cycle arrest program that prevents further expansion of the malignant cells. This is considered to be a failsafe mechanism by the tumor to prevent further "injury". Upon removal of the stress, this population promptly regains its proliferative nature, thereby leading to relapse and recurrence of the tumor.

Pancreatic adenocarcinoma is notorious for its resistance to therapy, metastasis and high rate of recurrence (www.cancer.gov). Studies from our laboratory show that a CD133+ population is associated with the aggressive biology of pancreatic adenocarcinoma ${ }^{2}$. While they are probably not a population that is responsible for the origin of pancreatic tumors, our previously published study

\section{(c) The Author(s) 2019}

(c) (i) Open Access This article is licensed under a Creative Commons Attribution 4.0 International License, which permits use, sharing, adaptation, distribution and reproduction c. in any medium or format, as long as you give appropriate credit to the original author(s) and the source, provide a link to the Creative Commons license, and indicate if changes were made. The images or other third party material in this article are included in the article's Creative Commons license, unless indicated otherwise in a credit line to the material. If material is not included in the article's Creative Commons license and your intended use is not permitted by statutory regulation or exceeds the permitted use, you will need to obtain permission directly from the copyright holder. To view a copy of this license, visit http://creativecommons.org/licenses/by/4.0/. 
definitely show that they are responsible for therapeutic resistance, tumor initiation at very low dilution as well as extreme metastasis $^{2-4}$. Our studies further show that this population is enriched upon nutritional deprivation, low dose chemotherapy as well as presence of hypoxia ${ }^{4-6}$. We and others have shown that CD133+ population are generally slow-cycling or quiescent ${ }^{2,7,8}$. This indicates that the cell cycle plays an active role in maintenance of this population in a quiescent and slow-cycling state.

Growth Arrest Specific 5 or GAS5, is a long non-coding RNA regulates cell cycle in a number of mammalian systems including several cancers ${ }^{9-12}$. It also mediates cell proliferation by regulating CDK6 activity ${ }^{13}$. Studies have also shown that GAS5 forms a positive feedback network with a number of genes involved in self-renewal like Sox2/ Oct4, making this long non-coding RNA (LncRNA) a critical player in induction and maintenance of the "stemness" state in a tumor ${ }^{14}$. GAS5 is further involved in regulation of human embryonic stem cell self-renewal by maintaining NODAL signaling ${ }^{15}$. Mechanistically, the effect of GAS5 on cell cycle is regulated by its interaction with the glucocorticoid receptor $(\mathrm{GR})^{16}$. GRs are nuclear receptor proteins that control cell proliferation via their effect on cell cycle ${ }^{17}$. GAS5 interacts with the activated GR preventing its association with the glucocorticoid response element (GRE) and consequently suppressing the transcription of target genes ${ }^{18}$. In pancreatic cancer, GAS5 has been shown to aid proliferation by regulating $\mathrm{CDK}^{13}{ }^{13}$ and also has important role in metastasis and chemoresistance ${ }^{19}$ all of which are important properties of CD133+ stem cells. However, the exact mechanism by which it plays a role in the growth and proliferation of CD133+ populations remains unanswered.

Studies from our laboratory have shown that the CD133 + population of cells is metabolically reprogrammed to be more dependent on glycolysis and has very low dependence on oxidative phosphorylation. Further, our studies have shown that this altered metabolic state promotes a "survival advantage" in this population by minimizing ROS accumulation ${ }^{4}$. Interestingly, while increased aerobic glycolysis is typically thought to be associated with proliferation, recent studies show that this metabolic activity may also be associated with other cellular functions as well ${ }^{20}$. Increased glucose uptake and metabolism is thus not necessarily required for robust growth of cells ${ }^{21,22}$. Literature also shows that glycolysis can be regulated by glucocorticoid receptor $(\mathrm{GR})^{23,24}$, and this can further affect cell cycle.

While CD133+ cells have been shown to be aggressive and therapy resistant, the molecular circuitry behind this still remains an enigma. It is also not clear why this population emerges in pancreatic tumors in response to microenvironmental as well as chemotherapeutic stress. In this study, we show for the first time that emergence of
CD133+ population coincides with upregulation of GAS5, that reprograms the cell cycle to slow proliferation by inhibiting GR mediated cell cycle control. The CD133+ population further routed metabolites like glucose to the pentose phosphate pathway, that were predominantly biosynthetic in spite of being quiescent in nature but did not use immediately for nucleic acid synthesis. Upon inhibiting GAS5, these cells were released from their growth arrest and restarted the nucleic acid synthesis and proliferation. Our study thus showed that GAS5 acts as a molecular rheostat for regulating quiescence and growth arrest in $\mathrm{CD} 133+$ population, that is responsible for aggressive biology of pancreatic tumors.

\section{Materials and methods \\ Cells and reagents}

Human cDNA CD133 expression plasmid (EX-Z0396M02) and empty vector plasmid (EX-NEG-M02) were obtained from GeneCopoeia. MIA PaCa-2, SU86.86 cells were obtained from ATCC and maintained as recommended. Stable MIA-derivatives (CD133Hi cells) were maintained in DMEM (Hyclone) containing 10\% fetal bovine serum. S2-VP10 cells were cultured in RPMI 1640 (Hyclone) supplemented with 10\% fetal bovine serum. Stable clones were selected and maintained in Geneticin (Invitrogen). Su86.86 with a CD133+ population of 28\%, KPC001 with a CD133+ population of $~ 10 \%$ and S2VP10 with $\sim 3-5 \%$ CD133+ cells are referred to as $\mathrm{CD} 133^{\mathrm{Hi}}$ cells in the study. Similarly, MIA-PaCa2 are referred to a CD $133^{\text {Lo }}$ cells since they have less that $0.01 \%$ CD133 + cells.

\section{Isolation and metabolic labeling of CD133+ tumor initiating cells from tumors}

The $\mathrm{CD} 133^{+}$population was separated from the mouse progenitor cells and other $\mathrm{CD} 133^{-}$cells using MACS separation (Miltenyi Biotech) following manufacturers protocol. Single cell suspension was generated from tumors in KPC mice according to $\mathrm{Li}$ et $\mathrm{al}^{25}$. Nonepithelial progenitor cells were removed using anti-CD31Biotin (BD Biosciences) and anti-CD45 Biotin (BD Bioscience) using MACS technique. The flowthrough free from the mouse progenitor cells was bound to anti-mouse $\mathrm{CD}_{133^{-}}$Microbeads for $10 \mathrm{~min}$ on ice and positively purified for $\mathrm{CD}_{133^{+}}$cells by MACS. The purity of separation was tested for each batch by performing a FACS analysis using Anti-CD133-PE antibody AC141 (Miltenyi Biotech).

\section{Metabolic labeling of CD133 + tumor initiating cells from tumors}

For labeling with ${ }^{13} \mathrm{C}$ glucose, isolated $\mathrm{CD} 133^{+}$cells were incubated in glucose free DMEM for $30 \mathrm{~min}$. Following incubation, the cells were transferred to a growth 
medium (DMEM) with ${ }^{13} \mathrm{C}$ glucose (final concentration $25 \mathrm{mM}$ ) and incubated further for $30 \mathrm{~min}$. Labeled cells were flash frozen and sent to University of Michigan Regional Comprehensive Metabolomic Resource Core for flux analysis. CD133- ${ }^{-}$cells were processed in parallel with ${ }^{12} \mathrm{C}$ glucose (final concentration $25 \mathrm{mM}$ ) added to DMEM.

\section{Cell cycle analysis}

Pancreatic cancer cells were plated in a 6-well plate and grown till 70-80\% confluent. Cells were harvested by trypsinization, washed and fixed in $70 \%$ cold ethanol at $4{ }^{\circ} \mathrm{C}$ for $30 \mathrm{~min}$ and treated with RNAse. A $100 \mu \mathrm{l}$ aliquot of this suspension was mixed with $100 \mu \mathrm{l}$ PI (Sigma Aldrich) reagent, incubated in dark for $30 \mathrm{~min}$ and then acquired on BD FACS Canto II. Cell cycle analysis was done by measuring total DNA content of the cells. For analysis in CD133+ vs CD133- cells, SU86.86 cells and/or KPC001 cells were sorted by staining with anti-CD133-PE in BD-FACS Aria. Sorted cells were grown for 2 weeks in growth media supplemented with $10 \%$ FBS. Once $70 \%$ confluent, analysis was done as described above.

For Pyronin Y staining, cells were grown or sorted as described above. For staining, cells were resuspended in $1 \mathrm{ml}$ cell culture medium containing $10 \mu \mathrm{g} / \mathrm{ml}$ Hoechst 33342 and incubated at $37^{\circ} \mathrm{C}$ for $45 \mathrm{~min}$. Five microliter of $100 \mu \mathrm{g} / \mathrm{ml}$ Pyronin Y (Sigma Aldrich) was added to the cells and incubated for another $15 \mathrm{~min}$. Cells were analyzed in BD FACS CantoII.

\section{Fluorescent in situ hybridization}

For FISH with GAS5 probe (Stellaris), three FFPE slides having pancreatic tumor sections were deparaffinized in $100 \%$ xylene for $10 \mathrm{~min}$ and additionally in fresh $100 \%$ xylene for $5 \mathrm{~min}$. Slides were then dipped in $100 \% \mathrm{EtOH}$ for $10 \mathrm{~min}$, again in fresh $100 \% \mathrm{EtOH}$ for $10 \mathrm{~min}$, and finally $70 \% \mathrm{EtOH}$ for at least $1 \mathrm{~h}$. Slides were incubated in 1x PBS for $5 \mathrm{~min}$ followed by 20 -min incubation with prewarmed proteinase $\mathrm{k}$ solution $(10 \mathrm{ug} / \mathrm{mL}$ proteinase $\mathrm{k}$ in $1 \mathrm{X}$ PBS warmed to $37^{\circ} \mathrm{C}$ ).

Hybridization was performed by immersing slides in wash buffer A ( $30 \mathrm{~mL}$ wash buffer A from Stellaris kit, $105 \mathrm{~mL} \mathrm{dH2O,} 15 \mathrm{~mL}$ deionized formamide) for $5 \mathrm{~min}$. Slides were removed from wash buffer A and excess was wiped off. $200 \mathrm{uL}$ hybridization buffer $(2 \mathrm{uL}$ Gas 5 probe stock $+200 \mathrm{uL}$ hybridization buffer) were added to each slide and a cover slip was applied to the top. Slides were incubated in the dark overnight at $37^{\circ} \mathrm{C}$ for no more than $16 \mathrm{~h}$.

Slides were immersed in wash buffer A to allow cover slip to detach and then incubated in the dark at $37^{\circ} \mathrm{C}$ for 30 min. ProLong Gold anti-fade reagent with DAPI was added to each slide and a new cover slip applied. Slides were stored in $4{ }^{\circ} \mathrm{C}$ in the dark and imaged with Leica fluorescent microscope.

\section{Statistics}

GraphPad Prism was used to determine the statistical significance for each study. Values are expressed as mean \pm SEM. All in vitro experiments were performed four times and significance between two samples was determined using the Student's paired $t$-test. Values were considered statistically significant when $p<0.05$.

\section{Results \\ $\mathrm{CD} 133^{\mathrm{Hi}}$ cells are non-proliferative and arrested in G0/G1 phase}

It is well known that "stem" cells are non-proliferative and quiescent. In our previous studies, we determined that pancreatic cancer cell lines like S2VP10, SU86.86 and KPC001 had higher number of CD133+ cells compared to MIAPACA2 and PanC01 ${ }^{2}$. We called the cell lines with increased $\mathrm{CD} 133+$ population, $\mathrm{CD} 133^{\mathrm{Hi}}$ while the cells with less CD133+ cells CD133 ${ }^{\mathrm{Lo}}$. Additionally, we also overexpressed CD133 constitutively in MIA-PACA2 cell line (that have negligible $\mathrm{CD} 133+$ population) to generate a MIA-CD $133^{\mathrm{Hi}}$ cell line ${ }^{3}$. To study if all cell lines with increased CD133 population were non-proliferative, we next tested the proliferation of cells with varied CD133 expression level. Our results showed that cell lines with increased CD133+ population like Su86.86 were less proliferative compared to cell lines with less CD133+ population (like MIA PaCa2) (Fig. 1a, b). This was similar to what was observed in isolated CD133+ cells from KPC tumors as well (Supplementary Fig. 1A). To study if this decreased proliferation was due to an arrest in cell cycle, we isolated CD133+ cells from KPC001 and SU86.86 cells and performed a cell cycle analysis by propidium iodide staining. Our studies showed that CD133+ cells (from both these cell lines) were arrested in G0/G1 phase with a significant increase in the G0 Phase cells, while the CD133 - cells progressed to G2/M phase regularly (Fig. 1c, d, Supplementary Fig. 1B). This was further confirmed by a Hoechst 33342/Pyronin Y staining. Generally, resting/ quiescent cells at G0 phase have lower levels of RNA compared with proliferating interphase cells (G1-S-G2-M phase). Pyronin Y intercalates both double stranded DNA and double stranded RNA. In the presence of DNAchelating fluorescent dye such as Hoechst 33342, interactions of Pyronin $\mathrm{Y}$ and DNA complex are disrupted and Pyronin Y mainly stains $\mathrm{RNA}^{26}$, allowing the quantification of RNA amount in a single cell level. Thus, resting cells can be identified by lower Pyronin Y for the same amount of DNA. Our results showed that CD133+ cells had an increase in low Pyronin stained population compared to CD133- cells in cells isolated from SU86.86 as well as KPC001 cell lines (Fig. 1e, Supplementary Fig. 1C). 

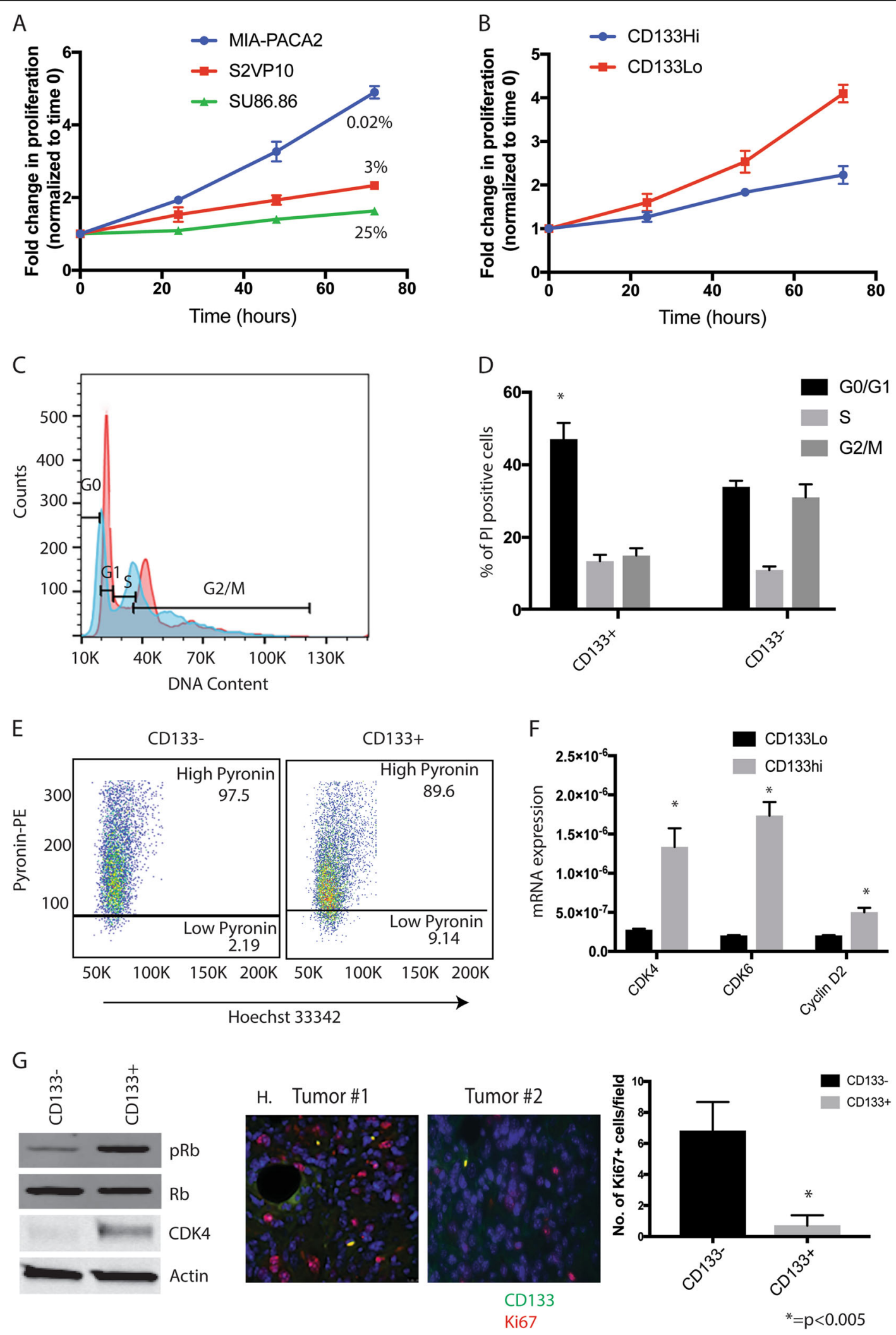

Fig. 1 (See legend on next page.) 
(see figure on previous page)

Fig. $1 \mathrm{CD} 133 \mathrm{Hi}$ cells are less proliferative and show growth arrest: WST-1 assay was done for cell proliferation. MIA-PACA2 cells with lowest CD133+ population showed highest proliferation while SU86.86 with high CD133+ population showed very little proliferation. Percent of CD133+ population is indicated on the curve (a). CD133 overexpressing cells (CD133Hi) also showed increased proliferation compared to CD133Lo cells (b). Cell cycle analysis showed CD133+ cells arrested at G0 state as seen by PI staining. Representative flow analysis (c) and bar graph (d) is shown. CD133+ cells had Low pyronin staining further confirming their G0/G1 arrest (e). Gene expression (f) and protein expression (g) of CDK4/6, Cyclin D2 and phosphorylation of Rb confirmed their arrest in this phase. Immunofluorescence of KPC tumors showed that the CD133+ cells (green) did not stain with Ki67 (red), a proliferative marker, further confirming that these cells were non-proliferative and quiescent (h).

To further understand the mechanisms of arrest we next analyzed the cell cycle regulator genes. Among these, cyclin dependent kinase 4/6 (CDK4/6) is important for G1 phase progression and is regulated by cyclin D. Cyclin D forms a complex with CDK4 to phosphorylate and inhibit retinoblastoma, $\mathrm{Rb}$ and regulates the $\mathrm{G} 1 / \mathrm{S}$ transition. Our results showed that in CD133hi cells had increased expression of CDK4, CDK6, and Cyclin D2 (Fig. 1f, g, Supplementary Fig. 1D) and increased phosphorylation of $\mathrm{Rb}$ (Fig. 1g). This indicates that in CD133+ cells, CDK4 expression and activity was higher than in CD133- cells leading to a G0/G1 arrest.

To further confirm these observations in vivo, we stained KPC001 derived tumors with CD133 and proliferation marker Ki-67. Our results showed that CD133+ cells typically did not stain with Ki67 (Fig. 1h), indicating their quiescence.

\section{Metabolites in quiescent $\mathrm{CD} 133^{\mathrm{Hi}}$ cells accumulate in biosynthetic pathways}

Previously published results from our laboratory showed that CD133+ pancreatic cancer cells had an altered metabolic pathway ${ }^{4}$. To further investigate this, we sorted pancreatic cancer cells isolated from KPC tumors into $\mathrm{CD} 133+$ and $\mathrm{CD} 133$ - populations and labeled them with ${ }^{13} \mathrm{C}_{6}$ glucose, and performed a flux analysis on them. Our results showed that in CD133+ cells, the glucose did not follow canonical glycolysis, but instead was routed through the pentose phosphate pathway, to produce lactate as observed by increased $13 \mathrm{C}$ labeled glucose $\left({ }^{13} \mathrm{C}_{6}\right.$ Glucose) in the pentose phosphate pathway intermediates (Fig. 2a, b). We next validated this by performing an activity assay for glucose 6 phosphate dehydrogenase (G6PD), the first enzyme in the pentose phosphate pathway in the CD133+ and CD133- population. As seen in the flux analysis, CD133+ cells had an increased G6PD activity indicating that in this population glucose was being routed through this metabolic pathway (Fig. 2c). To validate if this was a consequence of CD133 expression, we used MIA-CD133 ${ }^{\mathrm{Hi}}$ cells. As seen with the isolated CD133+ population, MIAPACA2 cells overexpressing CD133 (MIACD133 ${ }^{\mathrm{Hi}}$ ) cells showed an increased G6PD activity as well (Fig. 2d). Following this, G6PD activity was determined in other pancreatic cancer cell lines with variable expression of CD133. Our results showed that cells with high CD133 had increased G6PD activity compared to those with less CD133 population (Supplementary Fig. 2). We analyzed the mRNA expression of the genes involved in the pentose phosphate pathway. As seen in the flux analysis and the enzyme activity assay, the expression of the enzymes in pentose phosphate pathway were increased in $\mathrm{CD}_{133^{+}}$high compared to $\mathrm{CD} 133^{-}$ cells (Fig. 2e). Since pentose phosphate pathway predominantly contributes to the DNA synthesis, we studied the metabolic activity of this pathway in CD133+ cells. We used BrDU, a commonly used thymidine analog that is incorporated into the synthesizing DNA/RNA. Thus, an increase in $\mathrm{BrDU}$ incorporation over time would indicate an increase in nucleic acid synthesis. Our study showed that CD133+ cells had almost no BrDU incorporation (Fig. 2f), indicating a block in this pathway. This seemed paradoxical as we did not expect quiescent cells to have increased metabolic flux through the biosynthetic pathways like pentose phosphate pathway.

\section{Endogenous as well induced expression of CD133 in response to hypoxia, nutritional deprivation and chemotherapy induced stress leads to increased GAS5 expression along with suppression of proliferation}

It is known that availability of nutrients regulates cell proliferation by affecting gene transcription. In this context, CD133+ cells that are typically enriched during hypoxia in pancreatic cancer ${ }^{4-6}$ are nutritionally deprived. Studies have shown that both hypoxia as well as nutritional deprivation can result in quiescence in a cancer cell population leading to "senescence associated stemness" in a select population of tumor cells. These stressed cells overexpress growth arrest specific 5 or GAS5 gene ${ }^{16}$. Our analysis showed that cells having increased CD133 expression (whether overexpressed by pCMV-CD133 as in Fig. 3a) or endogenous (sorted from a pancreatic tumor) have significantly high GAS5 expression (Fig. 3b). Interestingly, when pancreatic cancer cell line MIAPACA2 was cultured under hypoxia or nutritional stress, GAS5 expression was also increased (Fig. 3c, d). Similar observation was made in other pancreatic cancer cell line as well (Supplementary Fig. 3A, B). Previously published results from our laboratory show that under all 


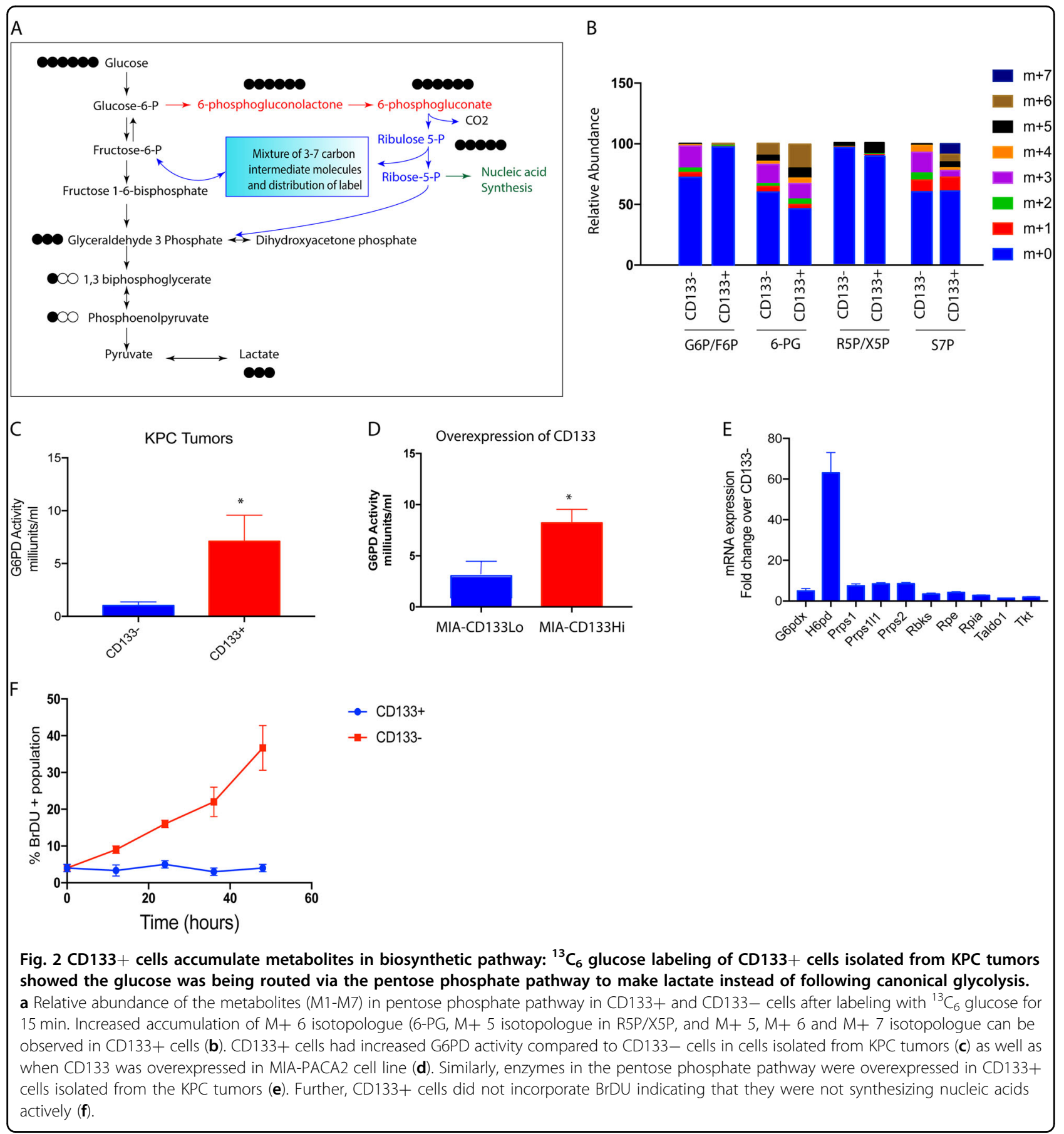

these conditions, CD133 expression was upregulated as well ${ }^{5,6}$. Our previously published work has shown that chemotherapy selected for treatment refractory CD133+ population ${ }^{6}$. To study if GAS5 was upregulated in pancreatic cancer cells under chemotherapy induced stress, we treated KPC001 cells with $100 \mathrm{nM}$ Gemcitabine for 7 days and analyzed the GAS5 and CD133 expression. As expected, these cells have extremely high expression of CD133. Along with this, the GAS5 expression was also high in these cells (Fig. 3e, Supplementary Fig. 3C). We next evaluated if modulation of CD133 affected GAS5 expression. Our results showed that silencing CD133 in S2VP10 cells using siCD133 decreased GAS5 expression (Fig. 3f). To study if increased GAS5 expression in CD133Hi cells contributed to its quiescence and growth arrest, we next inhibited GAS5 using siRNA in these cells and studied the effect on proliferation. Our study showed that inhibition of GAS5 increased proliferation in 


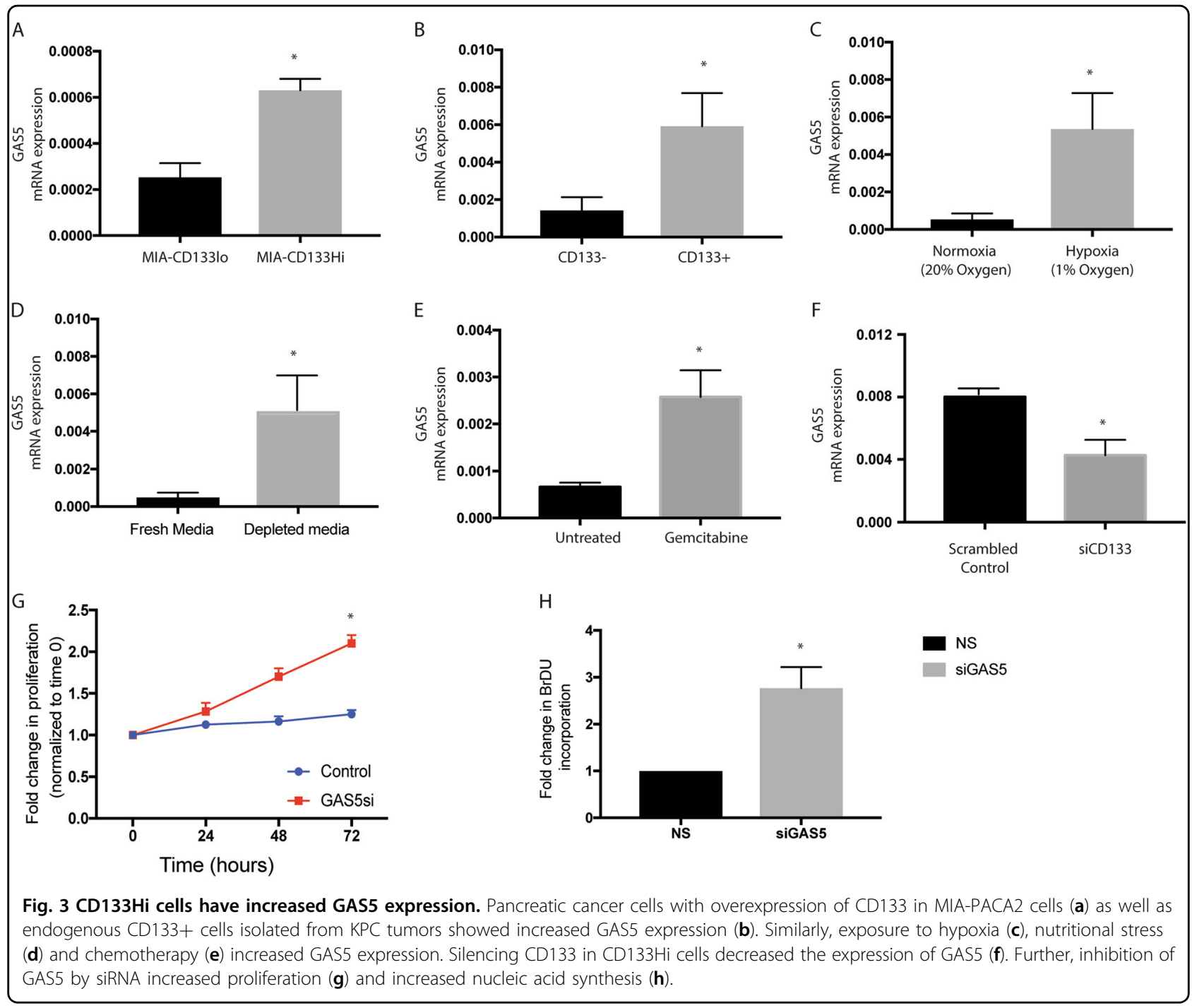

CD133Hi cells as seen by proliferation assays (Fig. 3g). To see if these cells now overcame the block in nucleic acid synthesis, we next evaluated their BrDU incorporation. Our study showed that along with increased proliferation, these cells had increased BrDU incorporation after $48 \mathrm{~h}$ of silencing GAS5 (Fig. 3h).

\section{GAS5 is overexpressed in pancreatic tumors}

Analysis of public database like Oncomine for GAS5 expression in pancreatic cancer showed that GAS5 was overexpressed in pancreatic tumors when compared to the normal tissues (Fig. 4a). Further, GAS5 was observed to be altered in $13 \%$ of the samples in the TCGA database hosted at www.cbioportal.org. Among these, mRNA amplification was found to be more prevalent than an alteration in the sequence (Fig. 4b). Additionally, GAS5 overexpression was more common in the pancreatic ductal adenocarcinoma and compared to the other types of pancreatic cancers (Fig. 4b). To visualize GAS5 in the pancreatic tumors, we next performed a fluorescent in situ hybridization with GAS5 on pancreatic cancer patient tumor slides as well as on implanted tumors with MIA-CD133 ${ }^{\mathrm{Hi}}$ cells. Our results confirmed that GAS5 was indeed overexpressed in pancreatic cancer (Fig. 4c, d). Further, expression of GAS5 increased as the tumor progressed (Fig. 4e).

\section{GAS5 expression and function was regulated by Sox 2 in CD133Hi pancreatic cancer cells}

We and others have shown that hypoxia increases expression and activity of self-renewal proteins like Sox2/ Oct4 and Nanog ${ }^{5}$. Similarly, nutritional deprivation as well as chemotherapy also induced expression of Sox2, Oct4, and Nanog expression ${ }^{6}$. Literature shows that GAS5 is regulated by Sox 2 and it forms a positive feedback loop with SOX2 and other transcription factors to regulate self-renewal in $\mathrm{mESCs}^{14,15}$. To see if in CD133+ 


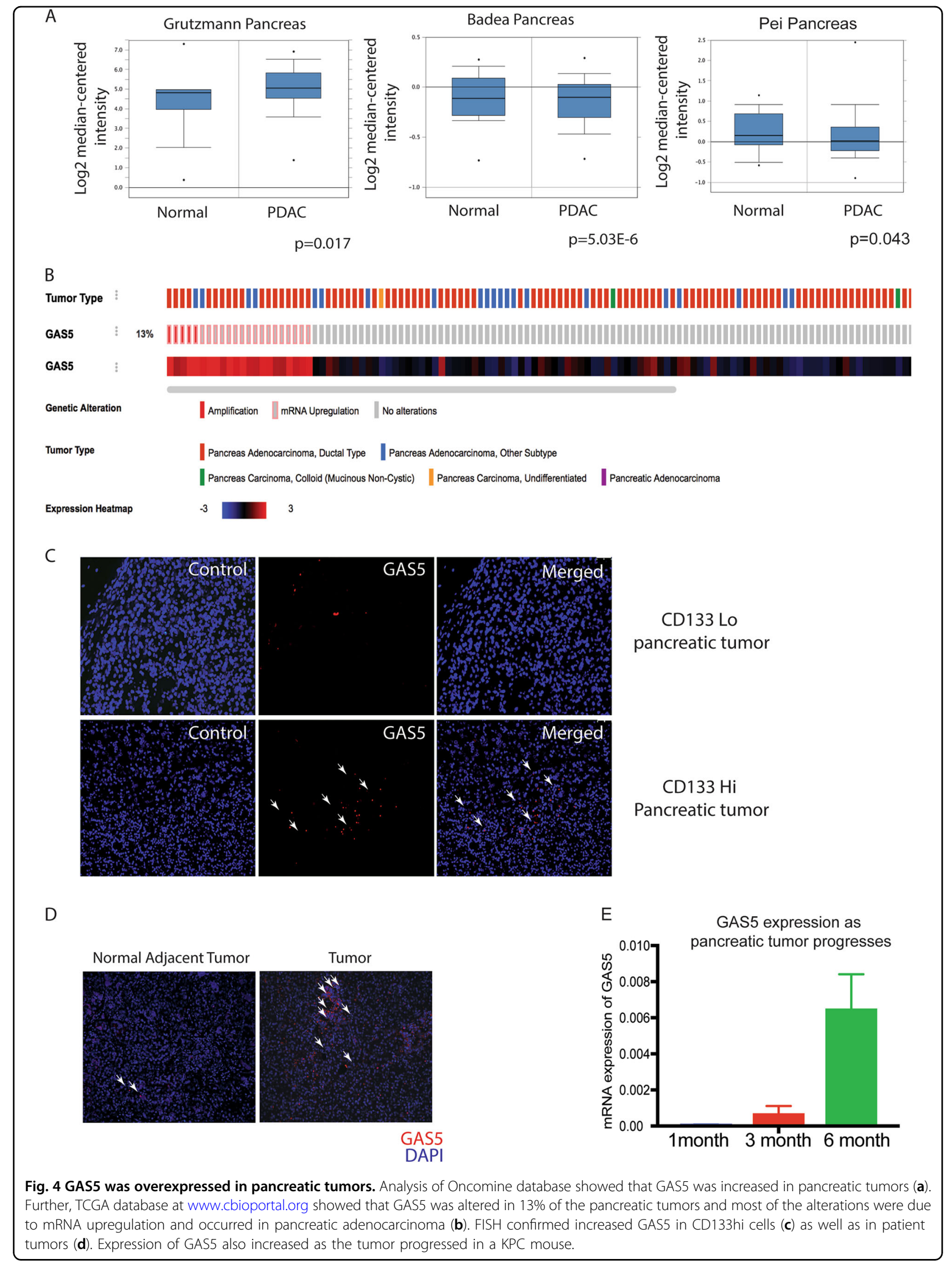



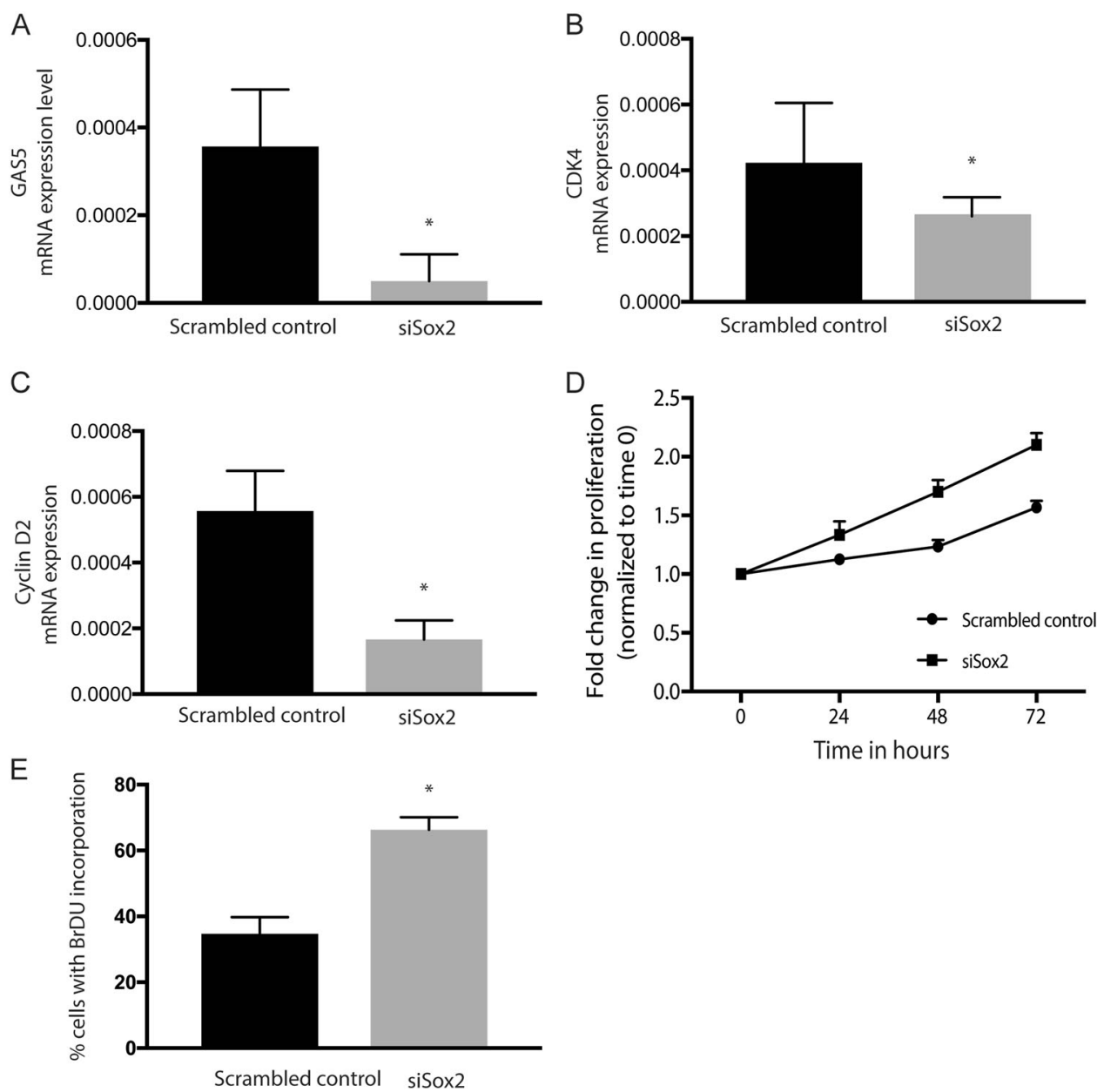

Fig. 5 Gas5 expression is regulated by Sox2. Inhibition of Sox2 by siRNA decreased GAS5 mRNA levels (a) along with expression of cell cycle genes CDK4 (b), Cyclin D2 (c) at the transcriptional level. Further, inhibition of Sox2 increased proliferation (d) along with increase in \% cells with BrDU incorporation indicating an increase in nucleic acid synthesis (e).

pancreatic cancer cells Sox2 regulated GAS5 mediated growth arrest, we next silenced Sox2 (with siRNA) and studied the expression of GAS5 in CD133 ${ }^{\mathrm{Hi}}$ cells. As seen in other cancers, inhibition of Sox 2 decreased GAS5 expression (Fig. 5a). Additionally, inhibition of Sox2 also decreased expression of CDK4/Cyclin D2 in the cells at the transcriptional (Fig. 5b, c). Since GAS5 was being downregulated with Sox2 inhibition, we hypothesized that this would release the "brake" from the proliferation arrest in the CD133+ cells and promote nucleic acid synthesis. Our study showed that indeed inhibition of Sox 2 led to increased proliferation of CD133Hi cells (Fig. 5d) as well as increased $\mathrm{BrDU}$ incorporation in $\mathrm{CD} 133 \mathrm{Hi}$ cells indicating an active nucleic acid synthesis (Fig. 5e).

GAS5 suppressed proliferation and downstream signaling in $\mathrm{CD} 133^{\mathrm{Hi}}$ cells by inhibiting GR mediated signaling

Previous studies have shown that CDK4/ Cyclin D axis can be regulated by glucocorticoid receptors (GR) mediated signaling ${ }^{17}$. Further, GR transcriptional activity is regulated by GAS5, which binds to the nuclear receptor GR and inhibits its activity. GR is an intracellular ligand dependent transcription factor that binds to GR elements (GRE) in the promoter region of select genes that regulate proliferation. To study if CD133Hi cells with high GAS5 expression consequently had low GR transcriptional activity, we next evaluated GR transcriptional activity using a dual luciferase reporter plasmid. Our study showed that cells with increased GAS5 expression had decreased GR transcriptional activity and inhibition of GAS5 by siRNA increased GR transcriptional activity (Fig. 6a). Since Sox2 was responsible for GAS5 transcription, we hypothesized silencing Sox2 will have a similar increase in GR transcriptional activity. Our results confirmed this hypothesis when Sox2 silencing increased GR transcriptional activity (Fig. 6a), establishing that Sox2-GAS5 negatively regulated GR activity. Since inhibition of GAS5 suppressed GR 


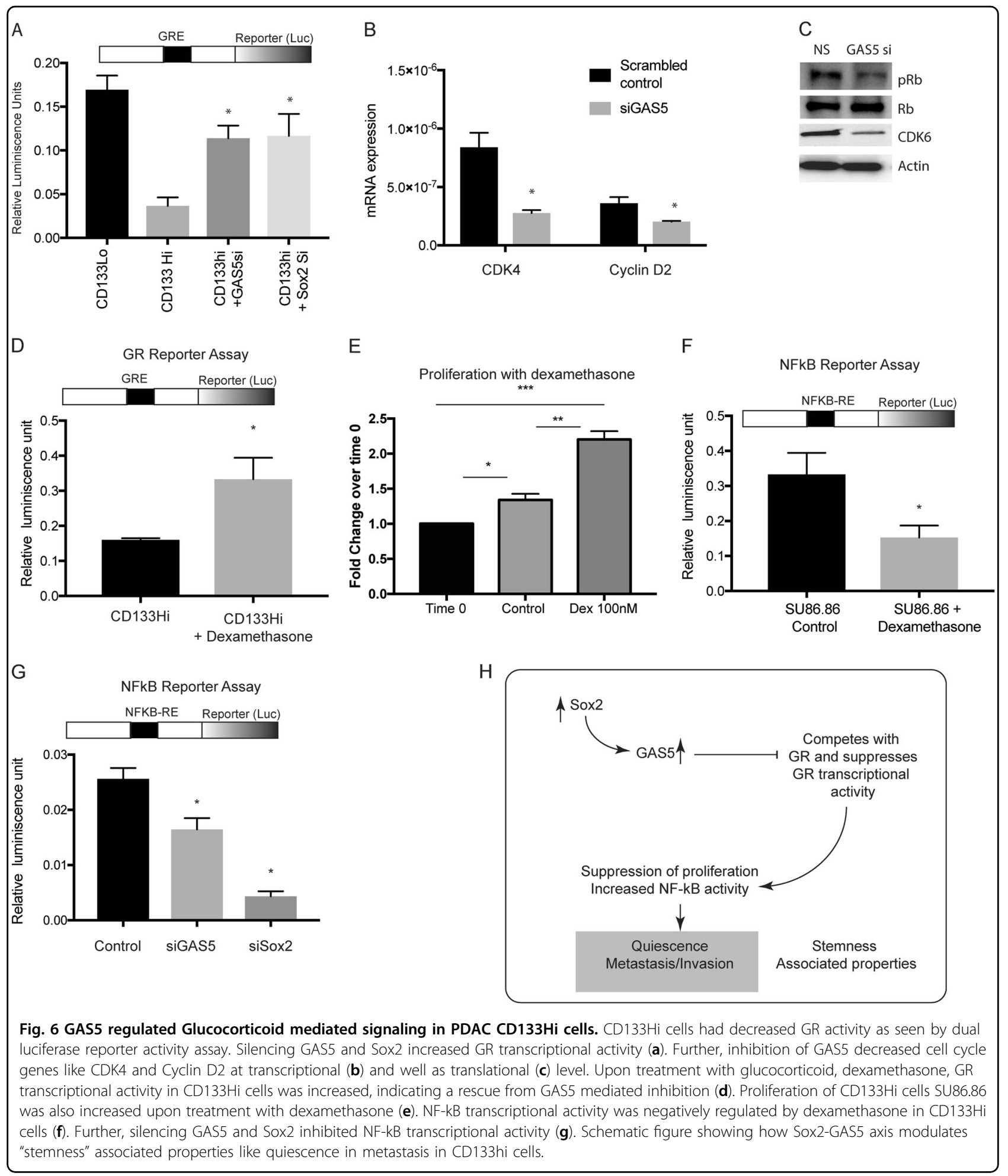

activity, we next tested the expression of CDK4/CyclinD expression along with $\mathrm{Rb}$ phosphorylation. Our results showed that as expected inhibition of GAS5 decreased expression of CDK4/CyclinD2 expression and decreased $\mathrm{Rb}$ phosphorylation (Fig. $6 \mathrm{~b}, \mathrm{c}$ ). This was consistent with the decreased proliferation observed with GAS5 inhibition in Fig. 3g.

To study if the GAS5 mediated growth arrest in CD133+ cells was mediated by GR, we stimulated $\mathrm{CD} 133^{\mathrm{Hi}}$ cells (that typically showed low GR activity) with dexamethasone 
(a GR agonist). Our results showed that stimulation of GR did not change GAS5 expression in these cells (Supplementary Fig 4A); however, the GR transcriptional activity was significantly increased (Fig. 6d). Consistent with this, the cells showed an increase in in proliferation when treated with dexamethasone (Fig. 6e). This showed that growth arrest in $\mathrm{CD} 133^{\text {hi }}$ cells was being regulated by GR mediated transcriptional activity.

Previous studies from our lab have shown that CD133+ cells are highly metastatic and this is regulated by the IL1$\mathrm{NF}-\mathrm{kB}$ axis ${ }^{3,27}$. GR has been known to negatively regulate $\mathrm{NF}-\mathrm{kB}^{28}$. This seemed consistent with our current observation that CD133Hi cells had increased GAS5 expression, had low GR transcriptional activity and subsequently high NF-kB activity in cells ${ }^{6}$. To study if GR modulation affected NF-kB activity, we next stimulated GR in SU86.86 cells (that have high CD133 and GAS5 expression). We hypothesized that upon stimulation of GR, the inhibition effect of GAS5 on GR will be overcome. Our results showed that upon stimulation of GR (with dexamethasone), NF-kB activity in these cells was decreased (Fig. 6f). Our results also showed that silencing Sox 2 or GAS5 decreased NF-kB reporter activity, further confirming the role of Sox2-GAS5 axis in mediating function of CD133Hi cells (Fig. 6g).

\section{Discussion}

Quiescence has been correlated with "cancer stemness" for a very long time. Early literature in the field reflected that the cancer stem cell (CSC) population was resistant to therapy because of their quiescence, as standard chemotherapy targeted rapidly proliferating cells $^{29-31}$. Quiescence properties in a stem cell can be controlled by intrinsic signaling pathways as well as influenced by the tumor microenvironment like hypoxia, interaction with stroma, as well as chemotherapy induced toxicity. Among intrinsic factors, the metabolic property of the cells as well as oncogenic signaling pathways like mTOR signaling play a vital role in determining quiescence ${ }^{32}$. Recent studies have shown that mitotically quiescent cancer stem cells in solid cancers like breast cancer tend to be more aggressive than the non- quiescent ones ${ }^{33,34}$. Consistent with this, previous results from our laboratory had identified a quiescent, rare population within pancreatic tumors represented by surface expression of CD133. This population correlated with invasiveness of the pancreatic cancer cells and tumors ${ }^{3,6,27}$ and was found to be responsible for initiating tumors at very low dilutions ${ }^{2}$. Further, results from our lab showed that this CD133+ population was enriched upon stress conditions like nutritional deprivation, hypoxia as well as chemotherapeutic stress ${ }^{4-6}$, and was identified as a "tumor initiating cell" even though it was not the cell of origin for pancreatic cancer.
Metabolic pathways in cancer have been recognized among the new "hallmarks of cancer" ${ }^{35}$. However, metabolic differences between actively proliferating and nonproliferating cells have not been studied extensively. Quiescent cells are typically known to favor catabolic metabolism compared to the proliferating cells ${ }^{36,37}$. However, within a tumor not all cells proliferate equally, and thus resulting in a metabolic heterogeneity within the tumor $^{38}$. Interestingly, metabolic flux analysis on isolated CD133+ cells from murine pancreatic tumor cells (KPC001) showed that in spite of being quiescent, a large number of metabolites were being fluxed through biosynthetic pathways like pentose phosphate pathway (Fig. 2). This appeared paradoxical, as according to literature, quiescent cells need to be reliant on catabolic rather than biosynthetic pathways in order to maintain homeostasis. Not a lot is known about pentose phosphate pathway (PPP) in context of CD133+ cancer stem cells. However, studies on pulmonary hypertension show that the key enzyme in this pathway G6PD, plays a critical role in hypoxia-induced CD133+ progenitor cells self-renewal ${ }^{39}$. Similarly, in sarcomatoid hepatocellular carcinoma showed co-expression of CD133 and G6PD had poor $\operatorname{prognosis}^{40}$.

Along with the observation that the CD133+ cells in a tumor had an active PPP, we also detected that irrespective of whether the cells have endogenously high expression of CD133 or CD133+ population was enriched by microenvironmental cues, these cells always had an overexpression of GAS5 (Fig. 4). Functionally, it has been reported that GAS5 IncRNA acts as a "riborepressor" for glucocorticoid receptor ${ }^{16}$. In cells that are arrested for growth, GAS5 is expressed abundantly and competes with glucocorticoids by binding to the DNA binding domain of glucocorticoid receptors (GR) thereby transcriptionally suppressing the GR regulated genes ${ }^{16}$.

Our study shows that in pancreatic cancer, CD133+ cells have a high expression of GAS5 (Fig. 3), which is regulated by the self-renewal transcription factor Sox2 (Fig. 5). Consistent with this, these cells are arrested for growth at the G0/G1 phase (Fig. 1). Additionally, these cells have low GR transcriptional activity (Fig. 6). Further, inhibition of GAS5 in CD133+ cells, results in increased proliferation, and a release from the cell cycle arrest (Fig. 4). Furthermore, inhibition of utilization of glucose by 2DG or inhibiting pentose phosphate pathway by DCA has no effect on GAS5 expression (Supplementary Fig. 3).

Recent studies have shown that a certain population of cells within the tumor may undergo chemotherapy (or stress) associated quiescence. This helps the population to tide over the unfavorable environment. Upon removal of the stress, these cells reactivate their proliferative phenotype by overcoming their growth arrest ${ }^{1}$. At this stage, the cells undergo a conversion to an aggressive growth 
phase that is extremely invasive and typically do not respond to therapy. Studies from our laboratory show that CD133+ population is enriched by microenvironmental "stressors" like hypoxia, associated nutritional deficiency as well as chemotherapy. This further increases their GAS5 levels and promotes quiescence. Additionally, GR signaling is known to negatively affect inflammatory pathways by regulating NF-kB. Our study shows that GAS5 mediated suppression of GR activity actually affects NF-kB signaling (Fig. 6g, h).

Thus, our study shows for the first time that in response to oncogenesis associated stress like hypoxia and nutritional deprivation, a small population of cells, represented by CD133 expression, tend to become quiescent by undergoing growth arrest and suppression of proliferation by overexpressing GAS5. GAS5 thus negatively regulated GR transcriptional activity contributing to this growth arrest. This regulation of "growth plasticity" in which metabolites could be stored in biosynthetic pathways during the quiescent phase of the cell cycle and not actively used provides this CD133+ population a survival advantage in which the cells can survive the unfavorable microenvironment by upregulating GAS5. Upon escaping the unfavorable condition, GAS5 expression is decreased and the "brakes" are released allowing CD133+ cells to draw upon the stored metabolites in biosynthetic pathways and jumpstart the processes required for active proliferation resulting in an aggressive tumor.

\section{Acknowledgements}

The authors would like to acknowledge Dr. Charles Burant and Dr. Maureen Kachman Michigan Regional Comprehensive Metabolomics Resource Core, University of Michigan for the metabolic flux analysis. The authors would also like to acknowledge the University of Miami Sylvester Cancer Center Flow Cytometry core for help with the flow cytometry based experiments. This study was funded NIH grant R01-CA184274 (to SB); Minneamrita Therapeutics LLC (to AKS), and the support from Sylvester Cancer Center (SB).

\section{Conflict of interest}

University of Minnesota has a patent for Minnelide, which has been licensed to Minneamrita Therapeutics, LLC. A.K.S. is the co-founder and the Chief Scientific Officer of this company. S.B. is a consultant with Minneamrita Therapeutics LLC and this relationship is managed by University of Miami. The remaining authors declare no conflict of interest.

\section{Publisher's note}

Springer Nature remains neutral with regard to jurisdictional claims in published maps and institutional affiliations.

Supplementary information accompanies this paper at (https://doi.org/ 10.1038/s41389-019-0177-4).

Received: 24 June 2019 Revised: 23 October 2019 Accepted: 28 October 2019

Published online: 18 November 2019

\footnotetext{
References

1. Milanovic, M. et al. Senescence-associated reprogramming promotes cancer stemness. Nature 553, 96-100 (2018).
}

2. Banerjee, S. et al. CD133+ tumor initiating cells in a syngenic murine model of pancreatic cancer respond to Minnelide. Clin. Cancer Res. 20, 2388-2399 (2014).

3. Nomura, A. et al. CD133 initiates tumors, induces epithelial-mesenchymal transition and increases metastasis in pancreatic cancer. Oncotarget $\mathbf{6}$, 8313-8322 (2015).

4. Nomura, A. et al. Microenvironment mediated alterations to metabolic pathways confer increased chemo-resistance in CD133+ tumor initiating cells. Oncotarget 7, 56324-56337 (2016).

5. McGinn, O. et al. Inhibition of hypoxic response decreases stemness and reduces tumorigenic signaling due to impaired assembly of HIF1 transcription complex in pancreatic cancer. Sci. Rep. 7, 7872 (2017).

6. Nomura, A. et al. Minnelide effectively eliminates CD133(+) side population in pancreatic cancer. Mol. Cancer 14, 200 (2015).

7. Francescangeli, F. et al. Proliferation state and polo-like kinase1 dependence of tumorigenic colon cancer cells. Stem Cells 30, 1819-1830 (2012).

8. Vincent, Z., Urakami, K., Maruyama, K., Yamaguchi, K. \& Kusuhara, M. CD133 - positive cancer stem cells from Colo205 human colon adenocarcinoma cell line show resistance to chemotherapy and display a specific metabolomic profile. Genes Cancer 5, 250-260 (2014).

9. Guo, X. et al. GAS5 Inhibits Gastric Cancer Cell Proliferation Partly by Modulating CDK6. Oncol. Res. Treat. 38, 362-366 (2015).

10. Liu, Y. et al. IncRNA GAS5 enhances G1 cell cycle arrest via binding to YBX1 to regulate p21 expression in stomach cancer. Sci. Rep. 5, 10159 (2015).

11. Liu, Z. et al. Downregulation of GAS5 promotes bladder cancer cell proliferation, partly by regulating CDK6. PLoS One 8, e73991 (2013).

12. Mourtada-Maarabouni, M., Hedge, V. L., Kirkham, L. \& Farzaneh, F. Growth arrest in human T-cells is controlled by the non-coding RNA growth-arrestspecific transcript 5 (GAS5). J. Cell Sci. 121, 939-946 (2008).

13. Lu, X. et al. Downregulation of gas 5 increases pancreatic cancer cell proliferation by regulating CDK6. Cell Tissue Res. 354, 891-896 (2013).

14. Tu, J. et al. Gas5 is an essential IncRNA regulator for self-renewal and pluripotency of mouse embryonic stem cells and induced pluripotent stem cells. Stem Cell Res. Ther. 9, 71 (2018).

15. $\mathrm{Xu}, \mathrm{C}$. et al. Long non-coding RNA GAS5 controls human embryonic stem cell self-renewal by maintaining NODAL signalling. Nat. Commun. 7, 13287 (2016).

16. Kino, T., Hurt, D. E., Ichijo, T., Nader, N. \& Chrousos, G. P. Noncoding RNA gas5 is a growth arrest- and starvation-associated repressor of the glucocorticoid receptor. Sci. Signal 3, ra8 (2010).

17. Rogatsky, I., Trowbridge, J. M. \& Garabedian, M. J. Glucocorticoid receptormediated cell cycle arrest is achieved through distinct cell-specific transcriptional regulatory mechanisms. Mol. Cell Biol. 17, 3181-3193 (1997).

18. Lucafo, M. et al. Long noncoding RNA GAS5: a novel marker involved in glucocorticoid response. Curr. Mol. Med. 15, 94-99 (2015).

19. Liu, B. et al. IncRNA GAS5 reverses emt and tumor stem cell-mediated gemcitabine resistance and metastasis by targeting miR-221/SOCS3 in pancreatic cancer. Mol. Ther. Nucleic. Acids. 13, 472-482 (2018). https://doi.org/ 10.1016/j.omtn.2018.09.026. Epub 6 Oct 2018.

20. Jones, W. \& Bianchi, K. Aerobic glycolysis: beyond proliferation. Front. Immunol. 6, 227 (2015).

21. Lemons, J. M. et al. Quiescent fibroblasts exhibit high metabolic activity. PLoS Biol. 8, e1000514 (2010).

22. Vander Heiden, M. G., Cantley, L. C. \& Thompson, C. B. Understanding the Warburg effect: the metabolic requirements of cell proliferation. Science $\mathbf{3 2 4}$, 1029-1033 (2009).

23. Dyczynski, M. et al. Metabolic reprogramming of acute lymphoblastic leukemia cells in response to glucocorticoid treatment. Cell Death Dis. $\mathbf{9}$, 846 (2018).

24. Lu, Y. et al. Glucocorticoid receptor promotes the function of myeloid-derived suppressor cells by suppressing HIF1alpha-dependent glycolysis. Cell Mol. Immunol. 15, 618-629 (2018).

25. Li, C., Lee, C. J. \& Simeone, D. M. Identification of human pancreatic cancer stem cells. Methods Mol. Biol. (Clifton, NJ) 568, 161-173 (2009).

26. Shapiro, H. M. Flow cytometric estimation of DNA and RNA content in intact cells stained with Hoechst 33342 and pyronin Y. Cytometry 2, 143-150 (1981).

27. Nomura, A. et al. NFkappaB-mediated invasiveness in CD133(+) pancreatic TICS is regulated by autocrine and paracrine activation of IL1 signaling. Mol. Cancer Res. 16, 162-172 (2018).

28. Yue, Q. et al. Downregulation of growth arrestspecific transcript 5 alleviates palmitic acidinduced myocardial inflammatory injury through the miR26a/ HMGB1/NFkappaB axis. Mol. Med. Rep. 18, 5742-5750 (2018). 
29. Adamski, V. et al. Dormant glioblastoma cells acquire stem cell characteristics and are differentially affected by Temozolomide and AT101 treatment. Oncotarget 8, 108064-108078 (2017).

30. Quayle, L. A., Ottewell, P. D. \& Holen, I. Chemotherapy resistance and stemness in mitotically quiescent human breast cancer cells identified by fluorescent dye retention. Clin. Exp. Metastasis 35, 831-846 (2018).

31. Touil, Y. et al. Colon cancer cells escape 5FU chemotherapy-induced cell death by entering stemness and quiescence associated with the c-Yes/YAP axis. Clin. Cancer Res. 20, 837-846 (2014).

32. Li, L. \& Bhatia, R. Stem cell quiescence. Clin. Cancer Res 17, 4936-4941 (2011).

33. Dembinski, J. L. \& Krauss, S. Characterization and functional analysis of a slow cycling stem cell-like subpopulation in pancreas adenocarcinoma. Clin. Exp. Metastasis 26, 611-623 (2009).

34. Pece, S. et al. Biological and molecular heterogeneity of breast cancers correlates with their cancer stem cell content. Cell 140, 62-73 (2010).
35. Hanahan, D. \& Weinberg, R. A. Hallmarks of cancer: the next generation. Cell 144, 646-674 (2011).

36. Cairns, R. A., Harris, I. S. \& Mak, T. W. Regulation of cancer cell metabolism. Nat Rev. Cancer 11, 85-95 (2011).

37. Wang, R. et al. The transcription factor Myc controls metabolic reprogramming upon T lymphocyte activation. Immunity 35, 871-882 (2011).

38. Mayers, J. R. \& Vander Heiden, M. G. Famine versus feast: understanding the metabolism of tumors in vivo. Trends Biochem Sci. 40, 130-140 (2015).

39. Chettimada, S. et al. Glucose-6-phosphate dehydrogenase plays a critical role in hypoxia-induced CD133+ progenitor cells self-renewal and stimulates their accumulation in the lungs of pulmonary hypertensive rats. Am. J. Physiol. Lung Cell Mol. Physiol. 307, L545-L556 (2014).

40. Yen, $\mathrm{C} . \mathrm{H}$. et al. Characterization of a new murine cell line of sarcomatoid hepatocellular carcinoma and its application for biomarker/therapy development. Sci. Rep. 7, 3052 (2017). 